\title{
Ne réinventons pas la roue, bâtissons des activités qualité sur l'acquis
}

\section{Christoph Bosshard}

Dr méd., vice-président de la FMH, responsable du département Données, démographie et qualité

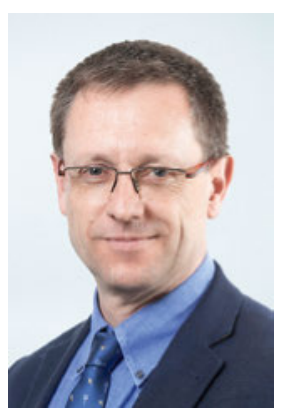

$\mathrm{Au}$ vu des débats actuels sur les primes et le financement de notre système de santé, nous n'aurions sûrement aucun mal à remplir tout un numéro du Bulletin des médecins suisses (BMS) à ce sujet. Si ces discussions animent, à juste titre, les politiques et le grand public, on a tendance à oublier les bénéfices rendus possibles grâce aux dépenses de santé - comme nous le rappelle la série d'articles publiée depuis début août dans le BMS.

C'est un autre thème tout aussi central que j'aimerais aborder ici, un thème qui fournit un point d'ancrage aux questions évoquées ci-dessus: je veux bien sûr parler de la qualité. Personne ne conteste que la qualité élevée des prestations fournies soit due selon le tarif. Cela se traduit d'une part dans le Code de déontologie de la FMH, et d'autre part, dans l'engagement volontaire des nombreuses organisations médicales qui ont signé la charte qualité de l'Académie suisse pour la qualité en médecine (ASQM). Depuis le lancement de la charte en octobre 2016, elles sont déjà 69 à avoir franchi le pas et à s'être ainsi engagées en toute liberté en faveur de la transparence et d'une prise de responsabilité dans ce domaine.

D'innombrables activités en faveur de la qualité, visibles ou moins visibles, sont depuis longtemps et seront toujours réalisées par le corps médical. Une des missions de l'ASQM est de faire connaître ces démarches et de permettre d'apprendre les uns des autres. De leur

\section{La mission de l'ASQM consiste à faire} connaître les activités existantes du corps médical en faveur de la qualité.

côté, les politiciens, les assureurs et l'administration sont toujours plus nombreux à exiger l'intensification des démarches en faveur de la qualité. Une exigence tout à fait justifiée, à condition de tenir compte de ce qui existe déjà et de construire sur cette base. Car les compétences requises ne se trouvent pas sur le coin d'un bureau, mais bien entre les mains des spécialistes et de leurs organisations professionnelles. De nombreux projets font preuve d'une volonté d'accomplir davantage dans ce domaine, mais ils échouent bien souvent faute de financement. Il revient au milieu politique de créer les conditions-cadres favorables pour y répondre. Dans sa stratégie Santé2020, la Confédération a formulé des objectifs et orientations qui doivent être mises en œuvre avec les acteurs de la santé. Pour trouver des solutions viables qui ne se perdent pas dans les méandres de la bureaucratie mais créent une véritable plus-value, le dialogue entre patients, assureurs, cantons, Confédération et fournisseurs de prestations est nécessaire. Un facteur de succès consiste en la délégation des instances responsables des organisations concernées dans les structures afin de susciter leur engagement. De ce dialogue naissent des stratégies de mise en œuvre et des

\section{Le dialogue entre patients, assureurs,} cantons, Confédération et fournisseurs de prestations est nécessaire pour trouver des solutions constructives.

mesures qui, à leur tour, peuvent être réalisées sous forme de processus transparents. Enfin au quotidien, les critères qualité doivent être réglés sous la forme de contrats-qualité entre assureurs, fournisseurs de prestations et cantons. A titre d'exemple, les trois critères qualité suivants peuvent être facilement appliqués et vérifiés dans le cadre de l'admission des médecins:

1. Activité médicale d'au moins trois ans dans un établissement de formation postgraduée reconnu dans la discipline demandée pour l'admission.

2. Compétences linguistiques attestées par un examen de langue passé en Suisse.

3. Présentation d'un diplôme ou d'une attestation de formation continue.

Afin de finalement connaître l'impact de toutes ces mesures, une recherche concomitante sur les services de santé est nécessaire. Le milieu politique a désormais la possibilité de mettre au point les conditions-cadres nécessaires lors du processus législatif actuel afin de renforcer la qualité et l'économicité (15.083). Je suis convaincu qu'ensemble, nous pourrons obtenir de nombreuses avancées dans l'intérêt de nos patients. Le corps médical est prêt à collaborer sur un pied d'égalité en tant que partenaire! 